Scientific Electronic Archives

Issue ID: Sci. Elec. Arch. Vol. 15 (1)

January 2022

DOI: http://dx.doi.org/10.36560/15120221490

Article link: https://sea.ufr.edu.br/SEA/article/view/1490

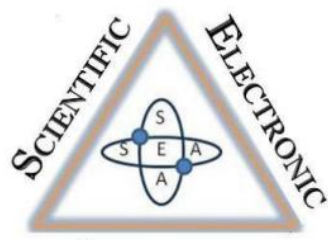

ArChives

ISSN 2316-9281

\title{
Fasceíte necrosante em um cão Pitbull - Relato de Caso
}

\author{
Necrotizing fasciitis in a Pitbull dog - Case Report
}

\author{
Corresponding author \\ Evelyn Barbosa Araújo \\ Universidade Federal de Mato Grosso \\ evelynbarbosaaraujo@gmail.com \\ Joaz Wellington Lopes \\ Universidade Federal de Mato Grosso \\ Luanna Ferreira Fasanelo Gomes \\ Universidade Federal de Mato Grosso
}

\begin{abstract}
Resumo. A fasceíte necrosante é uma infecção necrótica que pode acometer a fáscia superficial e profunda, pele e tecido subcutâneo, apresenta progressão rápida, podendo causar sepse se não tratada precocemente. Este artigo descreve um caso de fasceíte necrosante em um Pitbull macho de 4 anos. O animal apresentou edema de membro posterior com dor desproporcional e prostração. O tratamento prescrito foi firocoxib $5 \mathrm{mg} / \mathrm{kg}$ via oral, $24 / 24 \mathrm{~h}$ e dipirona $25 \mathrm{mg} / \mathrm{kg}$ via oral, $8 \mathrm{~h} / 8 \mathrm{~h}$. Devido a piora do quadro clínico o animal foi hospitalizado e veio a óbito. O diagnóstico se deu através da necropsia. O tratamento com desbridamento cirúrgico e antibiótico terapia sistêmica são preconizados nesses casos, a demora em sua instituição resulta em altas taxas de mortalidade.
\end{abstract}

Palavras-chaves Fasceíte necrosante, sepse, cães.

Abstract. Necrotizing fasciistis is a necrotic infection that can affect the superficial and deep fascia, skin and subcutaneous tissue, has rapid progression, and can cause sepsis if not treated early. This article describes a case of necrotizing fasciitis in a 4-year-old male Pitbull. The animal presented posterior limb edema with disproportionate pain and prostration. The prescribed treatment was firocoxib $5 \mathrm{~m} / \mathrm{kg}$ orally, $24 / 24 \mathrm{~h}$ and dipyrone $25 \mathrm{mg} / \mathrm{kg}$ orally, $8 / 8 \mathrm{~h}$. Due to the worsening of the clinical picture, the animal was hospitalzed and died. The diagnosis was made made through necropsy. Treatment with sugical debridement and antibiotic systemic therapy are recommended in these cases, the delay in your institution results in high mortality rates.

Keywords: Necrotizing fasciitis, sepsis, dogs.

\section{Introdução}

A fasceíte necrosante (FN) é uma infecção bacteriana grave, de rápida progressão, caracterizada por extensa desvascularização e necrose da fáscia profunda e superficial, podendo acometer também os tecidos associados (HAKKARAINEN et al., 2014; XU et al., 2019).

A FN é classificada de acordo com a sua etiologia (polimicrobiana ou monomicrobiana) (CHILDERS et al., 2002; GIULIANO et al., 1977; PUVANENDRAN, 2009). Estudo realizado por Wong et al. (2003) em humanos demonstrou que a fasceíte necrosante do tipo I (polimicrobiana) acomete com maior frequência indivíduos imunocomprometidos e a do tipo II (monomicrobiana) indivíduos sem predisposições. Em humanos a fasceíte necrosante do tipo 1 é a mais comum (MATTHEWS et al., 2019).

Os pacientes acometidos com NF podem apresentar sintomas de inflamação da pele, com dor desproporcional ao grau de inflamação, sepse e toxicidade sistêmica. Contudo também pode ser observado casos brandos sem acometimento incial da pele. (PUVANENDRAN, 2009; URSCHEL, 1999). Goh et. al, (2013) realizaram uma revisão de casos com 1463 pacientes humanos com fasceíte necrosante, no qual foi observado que os três principais sintomas iniciais foram: edema $(80,8 \%)$, 
dor $(79,0 \%)$ e eritema $(70,7 \%)$. Também foi observado que o diagnóstico inicial incorreto era comum devido as características não específicas.

Segundo as diretrizes práticas para diagnóstico e tratamento das infecções de pele e tecido mole em humanos (2014) o elemento mais importante no diagnóstico é o julgamento clínico associado ao desbridamento e exame visual direto dos tecidos, os quais - apresentam-se com coloração acinzentada e áreas de necrose, podendo estar presente exsudato fino acastanhado. O material preconizado para diagnóstico bacteriológico definitivo por cultura e coloração de gram é obtido por incisão exploratória e coleta de tecido profundo. Amostras obtidas de tecido superficial não são adequadas pois podem não conter os microrganismos presentes na lesão profunda (STEVENS et al., 2014).

$O$ diagnóstico e tratamento devem ser estabelecidos precocemente pois pode haver progressão para choque séptico e a morte do paciente (GOMES et al., 2016; URSCHEL, 1999). O tratamento preconizado é através do desbridamento precoce da ferida com excisão do tecido inviável e antibioticoterapia de amplo espectro intravenoso (PUVANENDRAN, 2009).

Devido a fasceíte necrosante ser um quadro atípico descrito em medicina veterinária, objetivou-se com esse estudo relatar um caso em um cão Pitbull atendido no Hospital Veterinário da Universidade Federal do Mato Grosso, Campus de Sinop.

\section{Relato de caso}

Um cão macho de 4 anos, $36,8 \mathrm{~kg}$, da raça Pitbull, foi atendido no Hospital Veterinário da UFMT, campus de Sinop com queixa de claudicação, edema do membro posterior direito, edema de bolsa escrotal (figura 1) e prostração há três dias. A tutora relatou não ter observado lesão de pele e o animal não apresentava histórico de trauma. Foi administrado pela tutora dipirona $1 \mathrm{mg} / \mathrm{kg}$ via oral, sendo observada melhora clínica.

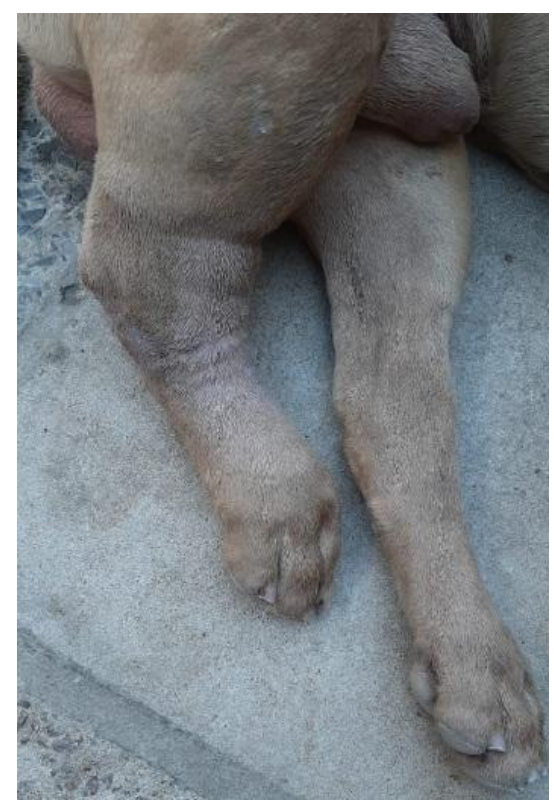

Figura 1. Membro posterior direito edemaciado. Fonte: arquivo disponibilizado pela tutora.

Ao exame físico o animal apresentou claudicação e dor ao manuseio do membro posterior direito, o qual que encontrava-se edemaciado e sem alteração na temperatura. Também encontrava-se edemaciada a bolsa escrotal. Foi observado aumento de linfonodo poplíteo direito, mucosas róseas, TPC $<2$, TR 38,9 C, frequência cardíaca 136 bpm, frequência respiratória $28 \mathrm{mpm}$.

Foi realizado hemograma e mensuração de creatinina , ALT e proteínas totais (quadro 1). Foi evidenciado linfopenia, anemia normocítica normocrômica, hiperproteinemia e valores de creatinina e ALT dentro da normalidade. Além de exame ultrassonográfico do membro acometido, que demonstrou somente a presença de edema do espaço subcutâneo. Foi prescrito firocoxib $5 \mathrm{mg} / \mathrm{kg}$ via oral $24 / 24 \mathrm{~h}$ e dipirona $25 \mathrm{mg} / \mathrm{kg}$ via oral $8 \mathrm{~h} / 8 \mathrm{~h}$.

$\mathrm{O}$ animal retornou após 2 dias com piora do quadro clínico, apresentando hiporexia, TR de 40,1 oC, mucosas hiperêmicas, taquipnéia, edema e aspecto eritematoso em região caudal ao tarso, tibial direita (figura 2), e também, em bolsa escrotal (figura 3). Foram realizados os exames de hemograma, proteínas totais, reticulócitos (quadro 1), mensuração de ALT, FA, creatinina e pesquisa de hemoparasitas. Foi demonstrado anemia normocítica normocrômica pouco regenerativa, linfopenia, desvio a esquerda regenerativo e aumento de FA $(341 \mathrm{U} / \mathrm{l})$ e creatinina $(1,9 \mathrm{mg} / \mathrm{dL})$. Também foi realizado teste rápido para detecção de anticorpos contra a Leishmaniose canina, este último tendo resultado negativo. 


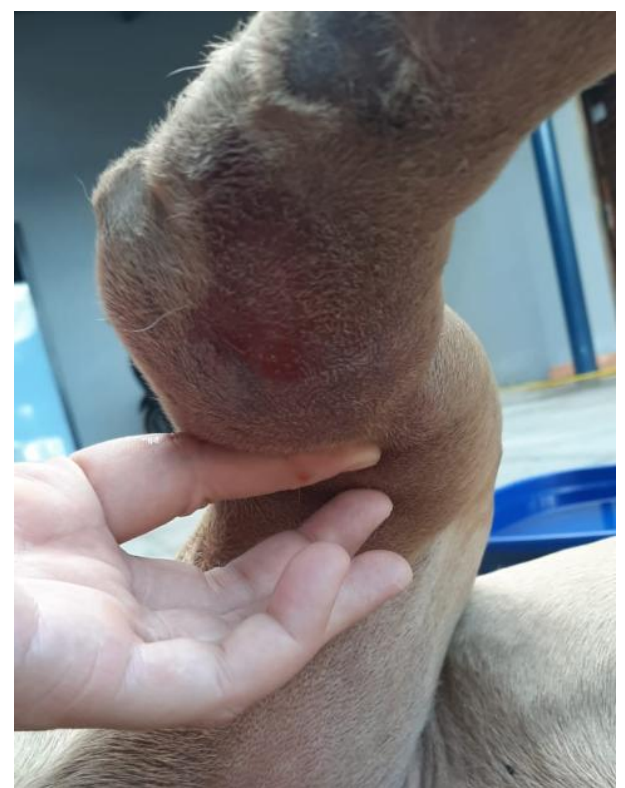

Figura 2. Edema e aspecto eritematoso em região caudal ao tarso e em região tíbial direita. Fonte: arquivo disponibilizado pela tutora.

Quadro 1. Hemograma realizado no dia $03 / 06 / 20$ e no dia 05/06/20, analisador eletrônico de células: BC 2800 VET (Mindray), contagem diferencial de leucócitos: esfregaço sanguíneo (100x) Hospital Veterinário da UFMT, Sinop.

\begin{tabular}{|lccc|}
\hline Hemograma & Valores Referência & Dia 03/06/20 & Dia 05/06/20 \\
\hline Hemácias $(/ \mu \mathrm{L})$ & 5.000 .000 a 8.500 .000 & 4.730 .000 & 5.330 .000 \\
Hemoglobina $(\mathrm{g} / \mathrm{dL})$ & 12 a 18 & 11.4 & 12,7 \\
Volume Globular $(\%)$ & 37 a 55 & 33 & 36,8 \\
VCM $(f \mathrm{~L})$ & 60 a 77 & 69.8 & 69,0 \\
CHCM $(\%)$ & 31 a 36 & 34.5 & 34,5 \\
Leucócitos Totais $(/ \mu \mathrm{L})$ & 6000 a 17000 & 15.300 & 11.800 \\
Neutrófilos segmentados $(/ \mu \mathrm{L})$ & 3000 a 11.500 & 11.475 & 9.086 \\
Neutrófilos Bastonetes $(/ \mu \mathrm{L})$ & 0 a 300 & 0 & 1.416 \\
Linfócitos $(/ \mu \mathrm{L})$ & 1.000 a 4.800 & 2.48 & 826 \\
Eosinófilos $(/ \mu \mathrm{L})$ & 100 a 1.250 & 153 & 118 \\
Monócitos $(/ \mu \mathrm{L})$ & 150 a 1.350 & 1.224 & 0 \\
Basófilos $(/ \mu \mathrm{L})$ & Raros & 0 & 354 \\
Plaquetas $(/ \mu \mathrm{L})$ & 200.00 a 500.000 & 243.000 & 338.000 \\
Reticulócitos & &.-- & 15.990 \\
Proteinas Totais $(\mathrm{g} / \mathrm{dL})$ & $5,5-8,0$ & 11,6 & 8,9 \\
\hline
\end{tabular}

Fonte: Arquivo disponibilizado pela tutora.

Devido ao agravo do quadro clínico e a não disponibilidade de hospitalização no HOVET-Sinop, o paciente foi encaminhado para outro centro veterinário. No dia 06/06/20 o animal veio a óbito, sendo congelado imediatamente após. O animal foi encaminhado para o setor de Patologia Animal do Hovet UFMT Sinop para a realização da necropsia, três dias após o óbito, obtendo-se diagnóstico de fasceíte necrosante.

$\mathrm{Na}$ necropsia foi observado macroscopicamente em região da articulação metacarpofalangeana laceração, com tecido com aspecto necrosado. No subcutâneo em região da articulação metacarpofalangeana apresentou edema multifocal moderado e musculatura hemorrágica difusa. Musculatura dos membros pélvicos e torácicos difusamente congestas e com áreas de moderada hemorragia multifocal. Também apresentou esplenomegalia, hepatomegalia, pulmão difusamente congesto e coração com moderada endocardiose em válvula mitral e bicúspide. 
Microscopicamente observou-se necrose acentuada de fibras musculares focalmente extensa, moderado edema de fibras musculares multifocal e leve infiltrado inflamatório de neutrófilos multifocal. Evidenciam-se ainda moderada necrose fibrinoide multifocal de parede de vasos sanguíneos associada à moderada trombose multifocal. Em coloração de GRAM, evidenciou-se estruturas compatíveis com bactérias cocoides de coloração roxa (Gram-Positiva). Também foi evidenciado pulmão com edema alveolar difuso acentuado e congestão difusa acentuada, fígado com congestão difusa acentuada e dilatação de sinusoides e necrose multifocal moderada de hepatócitos. Observou-se acentuada glomerulonefrite membranoproliferativa e glomeruloesclerose difusa e necrose tubular difusa aguda.

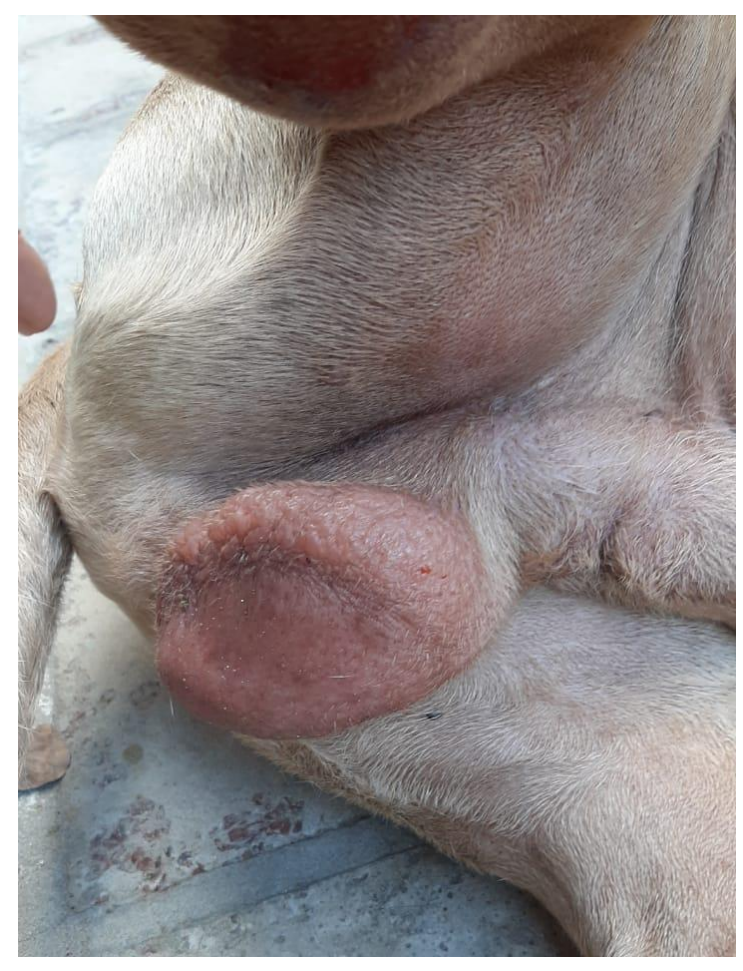

Figura 3. Bolsa escrotal edemaciada e eritematosa.

\section{Discussão}

São denominadas infecções necrosantes dos tecidos moles (INTM) doenças que afetam o tecido subcutâneo, pele, fáscia superficial e profunda, tecido muscular e tecido adiposo (LAGUNA et al., 2017).

As INTM têm como característica dor intensa e desproporcional a lesão cutânea (SHIROFF et. al, 2014) como observado no paciente relatado. Durante a primeira consulta foi identificado edema e não apoio do membro, também descritos em outros casos veterinários relatados de FN. (ABMA et al., 2013; NAIDOO et al., 2005; WIETHUCHTER, 2015). Nos cães os locais mais acometidos tem sido as extremidades (JENKINS et al., 2001). Rispoli (2020) relata que os sintomas mais frequentes observados em seu estudo em cães foram dor em 10 cães $(76,92 \%)$, prostação em 9 ( 69,23\%), taquipnéia em 8 $(61,64 \%)$, taquicardia febre e necrose subcutanea evidente em 7 ( 53,85\%).

Outro aspecto observado foi 0 não acometimento inicial da pele, característica descrita por Urshell (1999) em pacientes humanos e por Wiethuchter et., al. (2015) em cães. Existe a possibilidade de traumas com lesões não visíveis relacionados a contactantes como gatos, sendo o desencadeador do quadro de $\mathrm{FN}$, como relatado por Banovic et., al (2013) e que pode ter ocorrido neste caso pois o paciente convivia com 4 gatos.

Wang e colaboradores (2014) relatam que em humanos existe susceptibilidade correlacionada a pacientes com afecções crônicas e imunossuprimidos, o que não foi identificado neste caso. Rispoli (2020) relatou em seu estudo envolvendo 13 cães diagnosticados com $\mathrm{FN}$ a presença de doenças concomitantes em 6 cães $(46,15 \%)$ e em $7(53,85 \%)$ não havia comorbidades. $\mathrm{O}$ uso de AINEs foi avaliado quanto a correlação com o aumento do risco de complicações necrosantes, embora não tenha sido possível concluir tal hipótese, seu uso pode mascarar os sintomas e dificultar o diagnóstico. (SOUYRI et al., 2008).

A necrose da pele pode ser uma característica tardia o que dificulta a diferenciação inicial de infecções menos graves e o rápido diagnóstico (MALIK et al., 2010). Goh et., al. (2013) relatam em seu estudo de caso envolvendo 1463 pacientes humanos que os principais sintomas foram eritema, edema e dor, sendo estes inespecíficos, acarretaram diagnóstico incorreto em 
3/4 dos pacientes. Devido ao paciente não ter apresentado alterações inicias como eritema, febre e lesões necrosantes, a FN não foi apontada como diagnóstico diferencial.

Os exames hematológicos revelaram linfopenia no terceiro dia e desvio a esquerda regenerativo apenas no quinto dia. A maioria dos cães acometidos com FN apresentam leucocitose por neutrofilia e monocitose a partir do terceiro dia indicando processo toxinfecioso agudo. (RISPOLI, 2020). Processos inflamatórios podem acarretar o aumento das proteinas totais, como observado neste caso (Portas et al., 2021).

Os exames laboratoriais iniciais podem não conter alterações, mas com a progressão da doença são notados, como observado no paciente (XU et al., 2019). No terceiro e quinto dia o eritrograma revelou anemia normocítica normocrômica, e bioquímica sérica aumento de FA e creatinina corroborando como já relatado (RISPOLI 2020; XU et al., 2019).

Exames de imagem como ultrassonografia, raio-x e ressonância magnética são ferramentas muito utilizadas no diagnóstico de Fasceite necrosante sendo descrita em inúmeros casos. ( $X U$ et. al, 2019; RISPOLI, 2020; MAGUIRI et. al, 2015; ABMA et. al, 2013). O exame radiográfico pode ser observado presença de ar nos tecidos moles, mas a ausência desta característica não exclui o diagnóstico de FN (JENKINS et. al, 2010).

Foi realizado exame ultrassonográfico que revelou edema subcutâneo, mais evidente na região mediocaudal desde a coxa até o tarso, aumento de linfonodo poplíteo direito e estruturas tubulares sem presença de fluxo ao doppler, sugestivo de dilatação da vascularização linfática e edema da bolsa escrotal. Abma et., al, (2013) descreve aumento da ecogenicidade do subcutâneo da área afetada com inúmeras regiões hipoecoicas revezadas entre áreas pequenas com acúmulo de líquido.

Deve ser preconizado para o diagnóstico o julgamento clínico associado ao desbridamento e exame visual dos tecidos com posterior coleta de material dos tecidos profundos para cultivo microbiológico segundo as diretrizes práticas para diagnóstico e tratamento das infecções de pele e tecido mole em humanos (2014).

As alterações macroscópicas e microscópias são semelhantes as relatas por Rispoli (2020). Rispoli (2020) relata como os principais achados macroscópios subcutâneos e profundos da fáscia e musculatura a necrose, edema, exsudato serosanguinolento e hemorragia. Os principais diagnósticos morfológicos dos orgãos foram congestão e hemorragia pulmonar, edema pulmonar, microtrombose pulmonar, degeneração e necrose hepática centrolobular, degeneração e necrose tubular aguda renal. Foram observados ainda lesões vasculares como trombose, vasculite e embolos bacterianos.

As alterações hematológicas, os sinais clínicos como febre, taquipinéia, mucosas hiperêmicas e os achados anatomopatológicos sugerem a evolução da lesão da musculatura para o acometimento dos orgãos e choque séptico (CHAKRABORTY, BURNS 2020).

São relatados como principais agentes bacterianos correlacionados a FN em cães: Streptococcus $\beta$-hemolítico, Staphylococcus pseudointermedio, Staphylococcus haemolyticos, Staphylococcus hominis, Staphylococcus aureus, Estreptococcus canis, Escherichia Coli e Pasteurella Multocida (ABMA et al., 2013; BANOVIC et al., 2013; JENKINS et al., 2001; NAIDOO et al., 2005; PRESCOTT et al., 1997; JANOS et al., 2012; MAYER et al., 2012; WONG et al., 2005).

A coleta de material para exame histopatológico foi realizada pós mortem e não foi possível a identificação do agente.

Naidoo et. al, (2005) sugere iniciar o tratamento após o diagnóstico embasado na sintomatologia envolvendo febre alta, edema e dor desproporcional concomitantes. $\mathrm{O}$ tratamento consiste na remoção completa dos tecidos necrosados e antibióticoterapia sistemica de amplo espectro (SHARMA et al., 2012). O tratamento com antibióticos é essencial mas não substitui $O$ desbridamento cirurgico (BONNE; KADRI, 2017). O tempo entre o início dos sintomas e a instituição do tratamento adequado está associado ao prognóstico (FERNANDES 2011).

As alterações apresentadas pelo paciente a partir da segunda consulta, como taquipneia, mucosas hiperemicas, febre, taquicardia, juntamente com exames laboratoriais sugerem 0 quadro de sepse. Como não foi instituído o diagnóstico e tratamento precocemente o paciente veio a óbito como relatado em outros casos de FN(RISPOLI 2020).

O quadro clínico do paciente durou 7 dias até o óbito, Rispoli (2020) relata que os quadros clínicos observados em seu estudo duraram em média 5,85 +- 3,74 dias ( variando de 1 a 14 dias). A maioria dos animais (8/13) foi a óbito ou foi realizada eutanásia entre $0 \quad 4^{\circ}$ e $10^{\circ}$ dias de evolução.

\section{Conclusão}

A fasceíte necrosante deve ser incluída como diagnóstico diferencial principalmente em casos com associação de dor desproporcional, febre e edema, mesmo sem o acometimento da pele inicialmente. $O$ diagnóstico precoce associado ao desbridamento cirúrgico e antibiótico terapia sistêmica são imprescindíveis para um prognóstico favorável.

\section{Referências}

ABMA, E.; VANDENABEELE, S.; CAMPOS, M.; et al. Necrotizing fasciitis in a dog. Vlaams Diergeneeskundig Tijdschrift, v. 82, n. 3, p. 134142, 2013.

BANOVIC, F.; LINDER, K.; BOONE, A.; JENNINGS, 
S.; MURPHY, K. M. Cat scratch-induced Pasteurella multocida necrotizing cellulitis in a dog. Veterinary Dermatology, v. 24, n. 4, 2013.

BONNE, S. L.; KADRI, S. S. Evaluation and Management of Necrotizing Soft Tissue Infections. Infectious Disease Clinics of North America, v. 31, n. 3, p. 497-511, 2017. Elsevier Inc. Disponível em: <http://dx.doi.org/10.1016/j.idc.2017.05.011>.

CHAKRABORTY, R. K.; BURNS, B. Systemic Inflammatory Response Syndrome. In: StatPearls [Internet]. Treasure Island (FL): StatPearls Publishing; Janeiro de 2020 Disponível em: https://www.ncbi.nlm.nih.gov/books/NBK547669

CHILDERS, B. J.; POTYONDY, L. D.; NACHREINER, R.; et al. Necrotizing fasciitis: a fourteen-year retrospective study of 163 consecutive patients. The American surgeon, v. 68, n. 2, p. 10916, 2002. Disponível em: <http://www.ncbi.nlm.nih.gov/pubmed/11842952>. .

FERNANDES, C. et al. FASCEÍTE NECROTIZANTE PÓS-APENDICITE AGUDA. v. 24, p. 621-626, 2011.

GIULIANO, A.; LEWIS, F.; HADLEY, K.; BLAISDELL, F. W. Bacteriology of necrotizing fasciitis. The American Journal of Surgery, v. 134, $n$. 1, p. 52-57, 1977.

$\mathrm{GOH}$, T. et al. Early diagnosis of necrotizing fasciitis. British Journal of Surgery, v. 101, n. 1, p. 119-125, 2014.

GOMES, A. E. M.; SÁ, M. R.; MARQUES, M. DA C. G.; PINHEIRO, L. F. Aplicação do Índice LRINEC (Laboratory Risk Indicator for Necrotizing Fasciitis) na distinção entre infecções complicadas da pele e tecidos moles e infecções necrotizantes. Revista Portuguesa de Cirurgia, , n. 37, p. 9-16, 2016.

HAKKARAINEN, T. W.; KOPARI, N. M.; PHAM, T. N.; EVANS, H. L. Necrotizing soft tissue infections: Review and current concepts in treatment, systems of care, and outcomes. Current Problems in Surgery, v. 51, n. 8, p. 344-362, 2014.

JENKINS, C. M.; WINKLER, K.; RUDLOFF, E.; KIRBY, R. Necrotizing Fasciitis in a Dog. Journal of Veterinary Emergency and Critical Care, v. 11, n. 4, p. 299-305, 2001. Disponível em: $<$ http://doi.wiley.com/10.1111/j.1476-

4431.2001.tb00069.x>.

MAGUIRE, P. et al. The Successful Use of Negative-Pressure Wound Therapy in Two Cases of Canine Necrotizing Fasciitis. Journal of the American Animal Hospital Association, v. 51, n. 1, p. 43-48, 1 jan. 2015.

MALIK, V.; GADEPALLI, C.; AGRAWAL, S.; INKSTER, C.; LOBO, C. An algorithm for early diagnosis of cervicofacial necrotising fasciitis. European Archives of Oto-Rhino-Laryngology, v. 267, n. 8, p. 1169-1177, 2010. Disponível em: $<$ http://link.springer.com/10.1007/s00405-010-1248$5>$.

MATTHEWS, L.; GOODRICH, J. S.; WEBER, D. J.; BERGMAN, N. H.; MILLER, M. B. Closing the brief case: A Fatal Case of Necrotizing Fasciitis Due to Multidrug-Resistant Acinetobacter baumannii. Journal of Clinical Microbiology, v. 57, n. 7, p. 1-5, 2019.

MAYER, M. N.; RUBIN, J. E. Necrotizing fasciitis caused by methicillin-resistant Staphylococcus pseudintermedius at a previously irradiated site in a dog. Canadian Veterinary Journal, v. 53, n. 11, p. 1207-1210, 2012

NAIDOO, S. L.; CAMPBELL, D. L.; MILLER, L. M.; NICASTRO, A. Necrotizing Fasciitis: A Review. Journal of the American Animal Hospital Association, v. 41, n. 2, p. 104-109, 2005. Disponível em: <https://meridian.allenpress.com/jaaha/article/41/2/1 04/175954/Necrotizing-Fasciitis-A-Review>.

PORTAS, B. et al. Utilização da concentração plasmática de fibrinogênio e velocidade de hemossedimentação como parâmetro prognóstico em cães com leucocitose Use of plasma fibrinogen concentration and erythrocyte sedimentation rate as a prognostic parameter in dogs with leu. v. 2020, p. 1-9, 2021.

PRESCOTT, J. F.; MILLER, C. W.; MATHEWS, K. A.; YAGER, J. A.; DEWINTER, L. Update on canine streptococcal toxic shock syndrome and necrotizing fasciitis. Canadian Veterinary Journal, v. 38, n. 4, p. 241-242, 1997.

RISPOLI, V. F.P. . Investigação anatomopatológica de fascites necrotizantes leais em cães: há implicações em saúde pública?. 142f (Tese de Mestrado) - Faculdade de Medicina Veterinária e Zootecnia da Universidade de São Paulo, São Paulo, Brasil, 2020.

RUKSHINI PUVANENDRAN, J. C. M. H. AND S. P. Necrotizing fasciitis. Canadian family Physician, v. 55, n. 10, p. 981-987, 2009.

SHARMA, B.; SRIVASTAVA, M. K.; SRIVASTAVA, A.; SINGH, R. Canine streptococcal toxic shock syndrome associated with necrotizing fasciitis: An overview. Veterinary World, v. 5, n. 5, p. 311-319, 2012.

SHIROFF, A. M.; HERLITZ, G. N.; GRACIAS, V. H. Necrotizing Soft Tissue Infections. Journal of Intensive Care Medicine, v. 29, n. 3, p. 138-144, 29 maio 2014. 
SOUYRI, C.; OLIVIER, P.; GROLLEAU, S.; LAPEYRE-MESTRE, M. Severe necrotizing softtissue infections and nonsteroidal anti-inflammatory drugs. Clinical and Experimental Dermatology, v. 33, n. 3, p. 249-255, 2008.

STEVENS, D. L.; BISNO, A. L.; CHAMBERS, H. F.; et al. Practice guidelines for the diagnosis and management of skin and soft tissue infections: 2014 update by the infectious diseases society of America. Clinical Infectious Diseases, v. 59, n. 2, 2014.

URSCHEL, J. D. Classic diseases revisited: Necrotizing soft tissue infections. Postgraduate Medical Journal, v. 75, n. 889, p. 645-649, 1999. Disponível em:

<https://pmj.bmj.com/lookup/doi/10.1136/pgmj.75.88 9.645>.

VILLARREAL LAGUNA, A. G.; CAMPOS, D. R.; LAMBERT, M. M.; et al. Necrotizing Soft Tissue Infection in Dog - Case Report. Acta Veterinaria
Brasilica, v. 11, n. 4, p. 191-195, 2017.

WIETHUCHTER, C. F. E. T. T. D. C. V. No Title. CES CES Medicina Veterinaria y Zootecnia, v. 10, p. 64-70, 2015.

XU, L. Q.; ZHAO, X. X.; WANG, P. X.; YANG, J.; YANG, Y. M. Multidisciplinary treatment of a patient with necrotizing fasciitis caused by Staphylococcus aureus: A case report. World Journal of Clinical Cases, v. 7, n. 21, p. 3595-3602, 2019.

WANG, J.-M.; LIM, H.-K. Necrotizing fasciitis: eightyear experience and literature review. The Brazilian Journal of Infectious Diseases, v. 18, n. 2, p. 137143, mar. 2014.

WIETHUCHTER, C. F. E. T. T. D. C. V. No Title. CES CES Medicina Veterinaria y Zootecnia, v. 10, p. 64-70, 2015.

WONG, C.-H.; WANG, Y.-S. The diagnosis of necrotizing fasciitis. Current Opinion in Infectious Diseases, v. 18, n. 2, p. 101-106, abr. 2005. 